\title{
Molecular Anatomy of Plant Photoprotective Switches: The Sensitivity of PsbS to the Environment, Residue by Residue
}

\author{
Nicoletta Liguori, ${ }^{* \dagger \odot}$ Sara R. R. Campos, ${ }^{\ddagger}$ António M. Baptista, ${ }^{\ddagger 0}$ and Roberta Croce ${ }^{* \dagger}$ \\ ${ }^{\dagger}$ Department of Physics and Astronomy, Faculty of Sciences, Vrije Universiteit Amsterdam, De Boelelaan 1081, 1081 HV \\ Amsterdam, The Netherlands \\ ${ }^{\ddagger}$ Instituto de Tecnologia Química e Biológica António Xavier, Universidade Nova de Lisboa, 2780-157 Oeiras, Portugal
}

Supporting Information

\begin{abstract}
Under strong sunlight, plants avoid photooxidation by quenching the excess absorbed energy. Quenching is triggered by PsbS, a membrane protein that is activated and deactivated by the light-dependent $\mathrm{pH}$ changes in the thylakoid lumen. The mechanism of action of this protein is unknown, but it was suggested that several glutamates act as $\mathrm{pH}$ sensors. However, the $\mathrm{p} K_{\mathrm{a}}$ of glutamate is several $\mathrm{pH}$ units below the physiological values in the lumen. Thus, how can PsbS sense the $\mathrm{pH}$ of the lumen, and how does it respond to it? By applying a nonstandard molecular dynamics method that treats $\mathrm{pH}$ explicitly, we show that the lumen-exposed glutamates of PsbS have strongly shifted $\mathrm{p} K_{\mathrm{a}}$ values and that such shifts are crucial for the $\mathrm{pH}$ sensitivity in physiological conditions. We also demonstrate that protonation drives a systematic unfolding of a region key for protein-protein interactions, indicating that PsbS response to $\mathrm{pH}$ is a functional conformational switch.
\end{abstract}

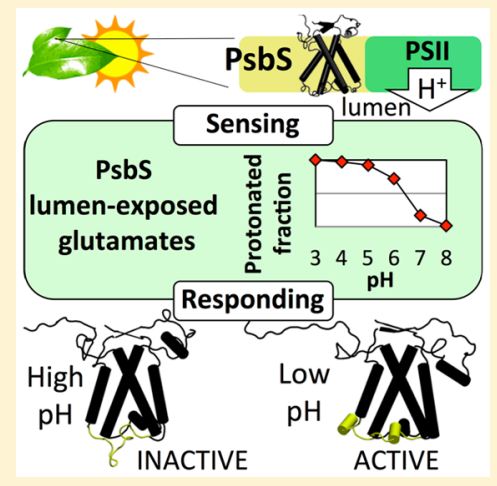

$\mathrm{P}$ lants avoid photooxidation by dissipating as heat the energy absorbed in excess in what is called energydependent quenching or $\mathrm{qE} .^{1}$ The light-harvesting complexes

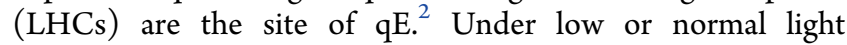
conditions, the LHCs transfer excitation energy to the core of photosystem II (PSII). Here, photochemistry is initiated and protons are pumped into the thylakoid lumen, an aqueous compartment enclosed by the photosynthetic membrane. Under high light, because of the increased rate of electron transfer, a proton gradient is established between the stromal and lumenal sides of the membrane, with the lumenal $\mathrm{pH}$ dropping from $\sim 7.5$ to $\sim 5.7( \pm 0.5){ }^{3}$ A membrane protein named PsbS is activated by the acidification of the lumen ${ }^{4,5}$ and triggers $\mathrm{qE}^{4,6}$

The essential role of $\mathrm{PsbS}$ in triggering $\mathrm{qE}$ in plants was discovered almost 20 years ago, ${ }^{7}$ and although several amino acid residues critical for its activity have been identified, its mechanism of action remains elusive. Also, although the crystal structure obtained at $\mathrm{pH} 5$ shows a dimeric complex, ${ }^{8}$ it has been suggested that the active form of PsbS (low $\mathrm{pH}$ ) is monomeric while the dimer is the dominating form at high $\mathrm{pH}$ values and is inactive. ${ }^{4-6,9,10}$ Furthermore, the overall effect of $\mathrm{pH}$ changes on the structure of PsbS is unknown and, to date, only the structure at $\mathrm{pH} 5$ has been resolved. ${ }^{8}$

To understand the structural basis of the $\mathrm{pH}$ sensitivity and the effect of $\mathrm{pH}$ changes on PsbS, we applied a state-of-the-art method known as constant-pH molecular dynamics (CpHMD). ${ }^{11,12} \mathrm{CpHMD}$ simulations are able to capture with atomistic resolution the time-dependent structural transitions of proteins at a given $\mathrm{pH}$. In brief, during each CpHMD trajectory, protonation states for the titratable residues of the protein are reset iteratively at given time intervals, depending on the local environment to which each residue is exposed and on the set $\mathrm{pH}$. To do so, CpHMD combines molecular dynamics (MD) simulations with continuum electrostatics (Poisson-Boltzmann or $\mathrm{PB}$ ) and Monte Carlo (MC) methods. Via the MD simulations, the protein is allowed to rearrange its structure in function of the protonation pattern at a given $\mathrm{pH}$. Via the $\mathrm{PB} / \mathrm{MC}$ calculations, the protonation pattern is then regularly updated throughout the MD simulation, allowing for the equilibration of the protein structure at the investigated $\mathrm{pH}$ value and the collection of quantitative information about the $\mathrm{pH}$ sensitivity of every residue. Although the calculation of $\mathrm{p} K_{\mathrm{a}}$ values is a challenging task, ${ }^{13}$ the CpHMD method has proven to be able to capture (i) the atypical $\mathrm{p} K_{\mathrm{a}}$ values that some residues exhibit depending on the local environment, ${ }^{14}$ (ii) the coupling between selected protein conformations and protonation states, ${ }^{12,15}$ and (iii) the entropy contribution of every possible protonation state at each titratable site. ${ }^{16}$

In this work, the dynamics of a monomer of PsbS in a simulated native-mimicking membrane has been investigated at 6 different $\mathrm{pH}$ values ranging from 3 to 8 . At each $\mathrm{pH}$ value investigated, 5 independent replicas were run for a total of 30 independent simulations and about $5 \mu \mathrm{s}$ of total simulated time. This titration allowed us to determine the $\mathrm{p} K_{\mathrm{a}}$ values of each protonable residue of $\mathrm{PsbS}$ and to identify the $\mathrm{pH}$ dependent conformational changes of the protein.

Received: February 14, 2019

Accepted: March 25, 2019

Published: March 25, 2019 

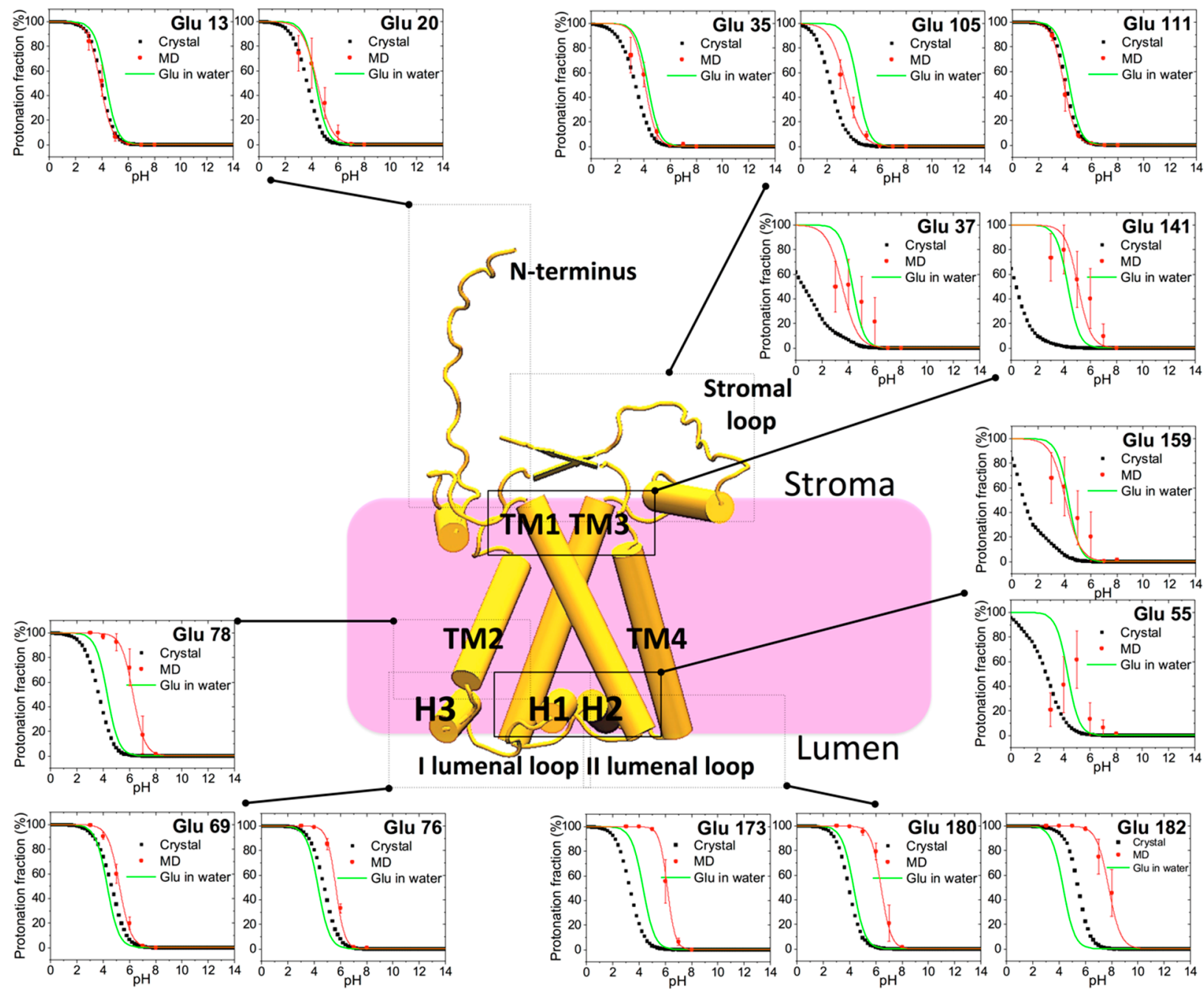

Figure 1. $\mathrm{pH}$ sensitivity of PsbS residue by residue: the case of Glu. Titration curves of selected Glu of PsbS exposed to different regions of the protein-membrane environment. The protein structure is reported in gold, and the light magenta box represents the membrane. Each red point in the graphs and the associated error bars were calculated, respectively, as an average and standard error over the 5 independent replicas equilibrated at each $\mathrm{pH}$ value (the first $100 \mathrm{~ns}$ of each replica was discarded from the analyses). The red solid curve in each graph represents the fit of the CpHMD data using the Hill approximation (see Methods in the Supporting Information).

In order to determine the sensitivity of $\mathrm{PsbS}$ to $\mathrm{pH}$, residue by residue, we calculated the frequency of protonation at a given $\mathrm{pH}$ for every titratable site over each $\mathrm{CpHMD}$ trajectory at equilibrium. By doing so, complete titration curves were reconstructed for the whole set of (protonable) residues. For comparison, the frequency of protonation at the different $\mathrm{pH}$ values was also computed on the crystal structure of $\mathrm{PsbS}^{8}$ embedded in the same equilibrated membrane-water system used for the CpHMD simulations (see Methods in the Supporting Information).

The titration curves for the whole set of titratable amino acids of PsbS are reported in Figure S1. Figures 1 and 2 focus on the glutamic acids (Glu) because several Glu residues have been proposed to be responsible for the $\mathrm{pH}$ sensitivity of the protein. ${ }^{17,18} \mathrm{We}$ found that all of the $\mathrm{p} K_{\mathrm{a}}$ values of the stromaexposed Glu (Glu13, Glu20, Glu35, Glu37, Glu105, Glu111, and Glu141) have a small shift (within one $\mathrm{pH}$ unit, Figure 2) from the $\mathrm{p} K_{\mathrm{a}}$ of Glu in water $\left(4.3,{ }^{19}\right.$ green curve in Figures 1 and $\mathrm{S} 1)$. This means that their $\mathrm{p} K_{\mathrm{a}}$ values are not strongly influenced by the protein environment and/or the lipid membrane. At striking variance with the stromal side, most of the lumenal exposed Glu have a $\mathrm{p} K_{\mathrm{a}}$ that is strongly shifted with respect to the value in water. These $\mathrm{p} K_{\mathrm{a}}$ shifts, up to 3.4 $\mathrm{pH}$ units in the case of Glu182 (Figure 2), make the majority of the lumenal exposed Glu (Glu69, Glu76, Glu78, Glu173, Glu180, and Glu182) sensitive to the range of physiological $\mathrm{pH}$ values that are critical for the activation of $\mathrm{qE}$. This means that when light saturates the photosynthetic machinery, the lumen-exposed Glu sense the associated change in $\mathrm{pH}$ allowing PsbS to respond to light fluctuations. The fact that multiple glutamates contribute to the sensitivity of PsbS is in agreement with experimental results ${ }^{17}$ showing that single or double mutations of Glu69, Glu78, Glu173, and Glu182 all resulted in plant lines with reduced $\mathrm{qE}$ levels. Our results show that all these residues have $\mathrm{p} K_{\mathrm{a}}$ values in the range of the thylakoid lumen. This is not the case for the other two lumen-exposed residues Glu55 and Glu159, which have a very low probability to be protonated at $\mathrm{pH}>5$. Interestingly, these residues were found to be essential for the proper folding and stability of PsbS. ${ }^{17}$ We found that the unprotonated form of these residues, which is present at all physiological $\mathrm{pH}$ values, preserves their role in the anchoring of the central transmembrane helices TM1 and TM3 via hydrogen bonds to the 


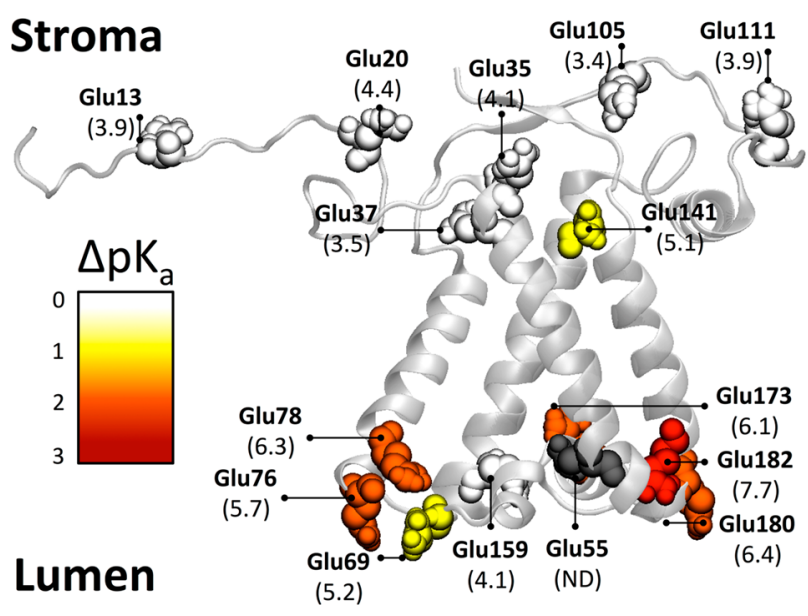

Figure 2. $\mathrm{p} K_{\mathrm{a}}$ shifts of PsbS Glu residues. The values reported in parentheses are the $\mathrm{p} K_{\mathrm{a}}$ of Glu computed via the CpHMD method by fitting the titration curves of the simulated structures in the Hill approximation (see Methods in the Supporting Information). The different colors associated with the Glu residues represent the difference between the computed $\mathrm{p} K_{\mathrm{a}}$ and the standard $\mathrm{p} K_{\mathrm{a}}$ value of Glu in water $\left(4.3^{19}\right) \cdot \mathrm{p} K_{\mathrm{a}}$ shifts below 0 and above 3 are colored in white and dark red, respectively. Glu residues are shown as van der Waals spheres. Glu55 (shown in gray) does not have a well-defined single midpoint $\mathrm{p} K_{\mathrm{a}}$ value (indicated as ND in the figure), as seen in its titration curve (Figure 1).

backbone of $\mathrm{H} 2$ and $\mathrm{H} 1,{ }^{8}$ respectively, thus stabilizing the protein (Figure S2A,B).

Large $\mathrm{p} K_{\mathrm{a}}$ shifts in proteins are well-studied, including in Glu residues, ${ }^{20,21}$ being a common benchmark for $\mathrm{p} K_{\mathrm{a}}$ prediction methods. ${ }^{13}$ They are caused by desolvation and/or electrostatics, both possibly modulated through $\mathrm{pH}$-dependent structural changes. Desolvation effects occur when a protein ionizable residue is not as exposed to the solvent as it would be when alone in aqueous solution, meaning that its charged form would be less stabilized by surrounding water dipoles and, therefore, the neutral-charged equilibrium would be shifted toward the neutral form. In the case of acidic groups (Glu, Asp, etc.), this would correspond to a $\mathrm{p} K_{\mathrm{a}}$ higher than when alone in solution. Therefore, if a Glu residue has a high $\mathrm{p} K_{\mathrm{a}}$ due only to desolvation, we would expect its solvent-accessible surface area (SASA) to be unusually low when the residue is neutral (the residue will always tend to get exposed when charged, even though that may happen only at unusually high $\mathrm{pH}$ ). Figure S3 shows that a reasonable relation is found between SASA and $\mathrm{p} K_{\mathrm{a}}$, except for Glu-37, Glu-141, and Glu-159. Therefore, desolvation seems to explain a part of the observed high $\mathrm{p} K_{\mathrm{a}}$ of Glu residues, but electrostatics must also be playing a role.

Electrostatic effects occur when a group experiences substantial electrostatic interactions from other charged groups, either because they are close to it or, if more distant, they can strongly interact through a region of low dielectric constant (e.g., the hydrophobic core of a protein or membrane). In the case of two acidic groups, such an interaction induces a positive $\mathrm{p} K_{\mathrm{a}}$ shift and, if they happen to titrate around the same $\mathrm{pH}$ region, the shift can be reciprocal, i.e., both groups will increase its $\mathrm{p} K_{\mathrm{a}}$. When these mutual shifts take place, the protonation of the two groups tends to be opposite, i.e, negatively correlated: their joint state is either charged/neutral or neutral/charged. Furthermore, when such strong interactions involve more than two residues, unexpected positive correlations may occur, with some pairs of groups exhibiting a charged/charged or neutral/neutral joint state, possibly associated with nonmonotonic "up-and-down" titration curves like those of Glu-55 and Glu-141, as experimentally observed in small compounds. ${ }^{22}$ The "outliers" in Figure S3, Glu-37, Glu-141, and Glu-159, are indeed found to engage in an extensive network of correlations with multiple residues, as illustrated in Figure S4. Therefore, the high $\mathrm{p} K_{\mathrm{a}}$ values of these Glu residues seem to be due to this network of electrostatic interactions.

It is important to note that, without the combination of continuum electrostatics and molecular dynamics (CpHMD), it would not have been possible to predict most of the $\mathrm{p} K_{\mathrm{a}}$ shifts, as it can be inferred from the titration curves computed on the crystal (Figures 1 and S1). This difference with the crystal structure strongly suggests that conformational dynamics are crucial in determining the $\mathrm{p} K_{\mathrm{a}}$ values of PsbS and, therefore, its functionality.

Similar to the stromal Glu, the aspartate residues, which are all present at the stromal side of PsbS, also showed $\mathrm{p} K_{\mathrm{a}}$ shifts of $<1$ unit with respect to the value in water (Figure S1), meaning that their $\mathrm{p} K_{\mathrm{a}}$ values remain outside of the physiologically relevant $\mathrm{pH}$ range. The $\mathrm{p} K_{\mathrm{a}}$ computed for the other titratable residues considered in this work (lysine and tyrosine) did not show deviation from the values estimated in water and were all above 8 (Figure S1).

We conclude that the ability of PsbS to sense the $\mathrm{pH}$ range relevant for the activation of $\mathrm{qE}$, which is $1-3 \mathrm{pH}$ units higher than the standard $\mathrm{p} K_{\mathrm{a}}$ of an acid residue, comes from the significant shift in $\mathrm{p} K_{\mathrm{a}}$ of its lumenal exposed Glu. This is particularly interesting considering that it has been proposed that the main role of $\mathrm{PsbS}$ is to raise the $\mathrm{pH}$ at which $\mathrm{qE}$ is triggered to a value which is physiologically attainable in the lumen. ${ }^{23}$

What is the response of PsbS to the different $\mathrm{pH}$ values? To answer this question, we characterized the conformational space of PsbS at the different $\mathrm{pH}$ values. In order to capture the $\mathrm{pH}$-dependent flexibility of PsbS, we computed the $\mathrm{pH}$ dependent root-mean-square fluctuation (RMSF) that is a measure of the conformational freedom of single residues at equilibrium. The sets of RMSFs per different $\mathrm{pH}$ values are shown in Figure S5. The RMSF analysis revealed that PsbS is characterized, at all $\mathrm{pH}$ values, by a rigid transmembrane region and by highly flexible solvent-exposed domains, namely, the termini and the first (I) and second (II) luminal loops (see Figure $3 \mathrm{~A}$ for a structural reference). To better distinguish which regions respond to $\mathrm{pH}$, we calculated the root-meansquare deviation (RMSD) per residue of the average protein structures at a given $\mathrm{pH}$ with respect to the average structure equilibrated at $\mathrm{pH} 5$, corresponding to the $\mathrm{pH}$ value at which the protein was crystallized. ${ }^{8}$ The different RMSD profiles are reported in Figure S5. The most substantial deviations were found at the termini and at the I- and II-lumen loops, where the highest RMSF was also found. This means that the apparent deviations from the structure at $\mathrm{pH} 5$ registered at these domains might originate from (i) an intrinsic disorder of these regions which results in high variance at all $\mathrm{pH}$ values or (ii) a real deviation from the structure at $\mathrm{pH} 5$.

To discriminate between these possibilities, we determined the equilibrated secondary structure of PsbS at the different $\mathrm{pH}$ values. In Figure S6, the frequency of the occurrence of specific types of local structures is reported for each protein domain. The possible types of local structure follow the DSSP 

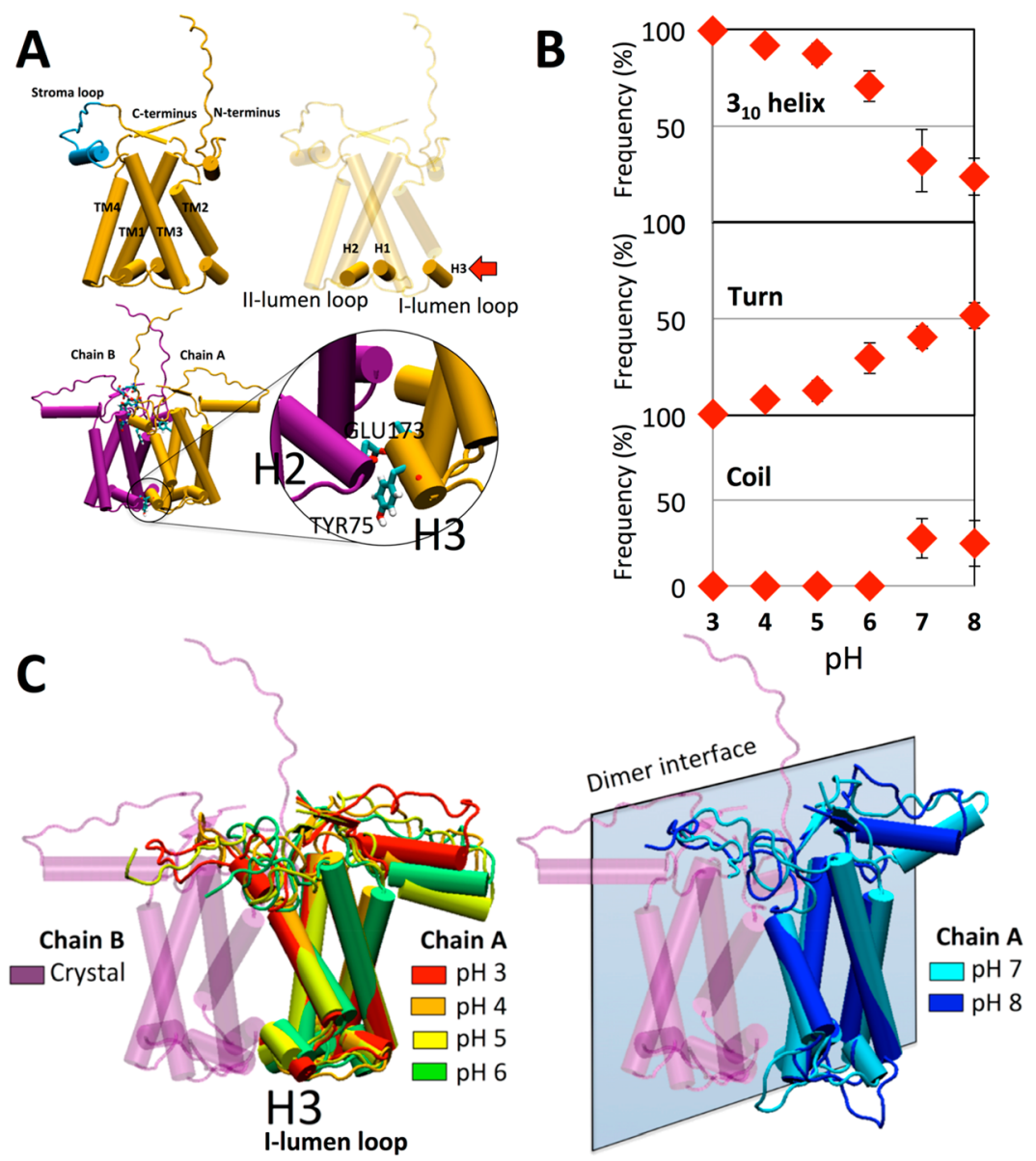

Figure 3. pH-dependent conformational change of PsbS. (A) Crystal structure of PsbS monomeric subunit (chain A, upper) and of PsbS dimer (lower). The amphipathic helices are indicated as well as the network of interactions between $\mathrm{H} 2$ and $\mathrm{H} 3$ in the inset. In cyan, the sequence reconstructed via the sequence homology model (see Methods in the Supporting Information) is reported. Chain B has been also reconstructed in the figure for the parts not resolved in the original structure. ${ }^{8}$ (B) $\mathrm{pH}$-dependent structure of H3. (C) Selected structures of PsbS monomer extracted from the $\mathrm{CpHMD}$ at different $\mathrm{pH}$ values as indicated in the legend and aligned on top of chain A to the crystal structure of PsbS dimer.

algorithm assignment (see Methods in the Supporting Information) and are $3_{10}$ helix, $\alpha$-helix, $\pi$-helix, $\beta$-bridge, $\beta$ sheet, turn, or coil. As reported in Figure S6, we found that, except for the I-lumen loop which contains the short amphipathic helixes $\mathrm{H} 1$ and $\mathrm{H} 3$ (Figure 3A), all other protein domains largely conserved at every investigated $\mathrm{pH}$ value (38) the type of secondary structure that they have in the crystal. ${ }^{8}$ Among these well-conserved domains are the C- and $\mathrm{N}$-terminus and the II-lumen loop containing helix $\mathrm{H} 2$, suggesting that their high RMSD (Figure S5) is due to an intrinsic disorder of these regions without a change of secondary structure. In the case of $\mathrm{H} 1$, instead, the simulations showed that the $\alpha$-helix present in the crystal structure had the tendency to change to either a $3_{10}$ helix or a turn, but without a clear $\mathrm{pH}$ dependence (values of $\alpha$-helix probability of 99\%, $95 \%, 73 \%, 62 \%, 93 \%$, and $84 \%$ in the $\mathrm{pH}$ range $3-8$, respectively).

What then is the response of PsbS to $\mathrm{pH}$ ? We systematically found that at high $\mathrm{pH}$ values $(\mathrm{pH} 7-8)$ the helical $\mathrm{H} 3$ domain switched to a lumen exposed loop (Figure 3C). In the crystal, $\mathrm{H} 3$ is hydrogen bonded via the backbones of Ile74 and Tyr75 to the short amphipathic helix $\mathrm{H} 2$ belonging to the other monomer in the PsbS crystal unit ${ }^{8}$ (Figure 3A). Fan et al. suggested that these hydrogen bonds are crucial in stabilizing the monomer-monomer interactions. ${ }^{8}$ The residue on $\mathrm{H} 2$ responsible for the hydrogen bond is Glu $173^{8}$ (Figure 3A), one of the putative $\mathrm{pH}$-sensitive sites of PsbS. ${ }^{17}$ Interestingly, as shown in Figure S6, protonation of Glu173 did not induce a conformational change in $\mathrm{H} 2$, and it conserved (together with the whole second (II) lumen loop) its structure at all $\mathrm{pH}$ values. On the other hand, high $\mathrm{pH}$ values ( 7 and 8 ) induced a complete unfolding of the amphipathic helix H3: this domain changed from a $3_{10}$ helix to a turn/coil (Figures 3B and S6). The representative conformations of $\mathrm{PsbS}$ at the different $\mathrm{pH}$ values are reported in Figure 3C.

What could be the functional effect on $\mathrm{qE}$ of the conformational switch of PsbS? The change in the structure of the $\mathrm{H} 3$ would result in the disruption of the ordered hydrogen bond network that anchors $\mathrm{H} 2$ to $\mathrm{H} 3$ in the dimer and weaken the interactions between monomers (Figure 3A,C and Supplementary Video 1). Our findings can explain the experimental results of Fan et al., who observed that the lumenal loops of the two PsbS monomers in the dimer are located at a larger distance at high $\mathrm{pH}^{8}$

Because at low $\mathrm{pH}$ PsbS is supposed to interact with other LHCs, ${ }^{6,9,24}$ we speculated that the creation of a $\mathrm{pH}$-sensitive docking mechanism through a network of tunable electrostatic interactions could be the basis of the mechanism of action of PsbS. Indeed, the alignment of any high-resolution structure of the homologous LHCs ${ }^{25,26}$ onto the central helices (the most 
conserved elements in the LHC family ${ }^{27}$ ) of one of the PsbS monomers in the dimeric crystal unit, results in dimers (Figure S7A-C) in which the PsbS H3 domain is facing the lumenal amphipathic helix of the other subunit, i.e., helix 4 for Lhcb and $\mathrm{H} 2$ for PsbS in the crystal. Such an organization (amphipathic helix 4 facing the amphipathic helix between the first and second transmembrane helices) is typical of dimeric LHCs in plants (Figure S7D). This would suggest that PsbS can stably dock to LHCs only at low $\mathrm{pH}$, when the $\mathrm{H} 3$ is structured (see Supplementary Video 2 for an example of the PsbS dimer at low $\mathrm{pH}$ ). In this respect, the presence of PsbS dimers in the crystal structure (resolved at $\mathrm{pH} \mathrm{5)}$ can be the result of the high capacity of PsbS to interact with other proteins at low $\mathrm{pH}$.

Overall, our work suggests that a conformational reorganization is the basis of the mechanism of action of PsbS in plants. This conformational reorganization is triggered by the protonation of multiple Glu residues exposed to the thylakoid lumen. We found that this is possible because the PsbS design adapted to match the range of physiological $\mathrm{pH}$ values of the thylakoid lumen by fine-tuning the $\mathrm{p} K_{\mathrm{a}}$ of its lumen-exposed residues.

\section{COMPUTATIONAL METHODS}

The methods are reported in the Supporting Information.

\section{ASSOCIATED CONTENT}

\section{S Supporting Information}

The Supporting Information is available free of charge on the ACS Publications website at DOI: 10.1021/acs.jpclett.9b00437.

PsbS monomer aligned to the chain $\mathrm{A}$ in the PsbS crystallized dimer and simulated here via $\mathrm{CpHMD}$ at $\mathrm{pH}$ 8 , showing the progressive loss of structure of its I-lumen loop at the interface between the two monomeric subunits, while the II-lumen loop maintains its structure (AVI)

PsbS monomer aligned to the chain $\mathrm{A}$ in the PsbS crystallized dimer and simulated here via $\mathrm{CpHMD}$ at $\mathrm{pH}$ 5 , showing the conservation of structure of its I- and IIlumen loops at the interface between the two monomeric subunits (AVI)

Fully detailed methods and supplementary figures (PDF)

\section{AUTHOR INFORMATION}

\section{Corresponding Authors}

*E-mail: n.liguori@vu.nl.

*E-mail: r.croce@vu.nl.

\section{ORCID $\odot$}

Nicoletta Liguori: 0000-0001-5695-4012

Sara R. R. Campos: 0000-0003-1020-6830

António M. Baptista: 0000-0002-7044-1210

\section{Notes}

The authors declare no competing financial interest.

\section{ACKNOWLEDGMENTS}

The authors are grateful to Vincenzo Mascoli and Mei Li for insightful discussions. This work is supported by The Netherlands Organization for Scientific Research (NWO), division Earth and Life science (ALW) via a Veni grant to N.L. and via a Vici grant to R.C.; by the ERC consolidator Grant 281341 (ASAP) to R.C.; by Project LISBOA-01-0145-FEDER007660 (Microbiologia Molecular, Estrutural e Celular) funded by FEDER funds through COMPETE2020 - Programa Operacional Competitividade e Internacionalização (POCI); and by national funds through FCT (Fundação para a Ciência e a Tecnologia). All the standard MD simulations in this work were carried out on the Dutch national e-infrastructure with the support of SURF Cooperative through a NWO grant to N.L. and R.C.

\section{REFERENCES}

(1) Müller, P.; Li, X.-P.; Niyogi, K. K. Non-Photochemical Quenching. A Response to Excess Light Energy. Plant Physiol. 2001, 125, 1558-1566.

(2) Ruban, A. V.; Johnson, M. P.; Duffy, C. D. P. The Photoprotective Molecular Switch in the Photosystem II Antenna. Biochim. Biophys. Acta, Bioenerg. 2012, 1817, 167-181.

(3) Takizawa, K.; Cruz, J. A.; Kanazawa, A.; Kramer, D. M. The Thylakoid Proton Motive Force in Vivo. Quantitative, Non-Invasive Probes, Energetics, and Regulatory Consequences of Light-Induced Pmf. Biochim. Biophys. Acta, Bioenerg. 2007, 1767, 1233-1244.

(4) Li, X.-P.; Müller-Moulé, P.; Gilmore, A. M.; Niyogi, K. K. PsbSDependent Enhancement of Feedback de-Excitation Protects Photosystem II from Photoinhibition. Proc. Natl. Acad. Sci. U. S. A. 2002, 99, $15222-15227$.

(5) Bergantino, E.; Segalla, A.; Brunetta, A.; Teardo, E.; Rigoni, F.; Giacometti, G. M.; Szabò, I. Light- and pH-Dependent Structural Changes in the PsbS Subunit of Photosystem II. Proc. Natl. Acad. Sci. U. S. A. 2003, 100, 15265-15270.

(6) Wilk, L.; Grunwald, M.; Liao, P.-N.; Walla, P. J.; Kühlbrandt, W. Direct Interaction of the Major Light-Harvesting Complex II and PsbS in Nonphotochemical Quenching. Proc. Natl. Acad. Sci. U. S. A. 2013, 110, 5452-5456.

(7) Li, X. P.; Björkman, O.; Shih, C.; Grossman, a R.; Rosenquist, M.; Jansson, S.; Niyogi, K. K. A Pigment-Binding Protein Essential for Regulation of Photosynthetic Light Harvesting. Nature 2000, 403, 391-395.

(8) Fan, M.; Li, M.; Liu, Z.; Cao, P.; Pan, X.; Zhang, H.; Zhao, X.; Zhang, J.; Chang, W. Crystal Structures of the PsbS Protein Essential for Photoprotection in Plants. Nat. Struct. Mol. Biol. 2015, 22, 729735 .

(9) Bonente, G.; Howes, B. D.; Caffarri, S.; Smulevich, G.; Bassi, R. Interactions between the Photosystem II Subunit PsbS and Xanthophylls Studied in Vivo and in Vitro. J. Biol. Chem. 2008, 283, 8434-8445.

(10) Krishnan, M.; Moolenaar, G. F.; Gupta, K. B. S. S.; Goosen, N.; Pandit, A. Large-Scale in Vitro Production, Refolding and Dimerization of PsbS in Different Microenvironments. Sci. Rep. 2017, 7, 15200.

(11) Baptista, A. M.; Teixeira, V. H.; Soares, C. M. Constant-pH Molecular Dynamics Using Stochastic Titration. J. Chem. Phys. 2002, $117,4184-4200$.

(12) Machuqueiro, M.; Baptista, A. M. Constant-pH Molecular Dynamics with Ionic Strength Effects: Protonation-Conformation Coupling in Decalysine. J. Phys. Chem. B 2006, 110, 2927-2933.

(13) Alexov, E.; Mehler, E. L.; Baker, N.; Baptista, A. M.; Huang, Y.; Milletti, F.; Nielsen, J. E.; Farrell, D.; Carstensen, T.; Olsson, M. H. M. Progress in the Prediction of $\mathrm{pKa}$ Values in Proteins. Proteins: Struct., Funct., Genet. 2011, 79, 3260-3275.

(14) Machuqueiro, M.; Baptista, A. M. Is the Prediction of pKa Values by Constant-pH Molecular Dynamics Being Hindered by Inherited Problems? Proteins: Struct., Funct., Genet. 2011, 79, 34373447.

(15) Campos, S. R. R.; Machuqueiro, M.; Baptista, A. M. Constant$\mathrm{pH}$ Molecular Dynamics Simulations Reveal a $\beta$-Rich Form of the Human Prion Protein. J. Phys. Chem. B 2010, 114, 12692-12700. 
(16) Baptista, A. M.; Soares, C. M. Some Theoretical and Computational Aspects of the Inclusion of Proton Isomerism in the Protonation Equilibrium of Proteins. J. Phys. Chem. B 2001, 105, 293-309.

(17) Li, X.-P.; Phippard, A.; Pasari, J.; Niyogi, K. K. Structurefunction Analysis of Photosystem II Subunit S (PsbS) in Vivo. Funct. Plant Biol. 2002, 29, 1131-1139.

(18) Li, X.-P.; Gilmore, A. M.; Caffarri, S.; Bassi, R.; Golan, T.; Kramer, D.; Niyogi, K. K. Regulation of Photosynthetic Light Harvesting Involves Intrathylakoid Lumen $\mathrm{pH}$ Sensing by the PsbS Protein. J. Biol. Chem. 2004, 279, 22866-22874.

(19) Grimsley, G. R.; Scholtz, J. M.; Pace, C. N. A Summary of the Measured $\mathrm{pK}$ Values of the Ionizable Groups in Folded Proteins. Protein Sci. 2009, 18, 247-251.

(20) Isom, D. G.; Cannon, B. R.; Castañeda, C. A.; Robinson, A.; García-Moreno E., B. High Tolerance for Ionizable Residues in the Hydrophobic Interior of Proteins. Proc. Natl. Acad. Sci. U. S. A. 2008, 105, 17784-17788.

(21) Isom, D. G.; Castañeda, C. A.; Cannon, B. R.; Velu, P. D.; García-Moreno E., B. Charges in the Hydrophobic Interior of Proteins. Proc. Natl. Acad. Sci. U. S. A. 2010, 107, 16096-16100.

(22) Submeier, J. L.; Reilley, C. N. Nuclear Magnetic Resonance Studies of Protonation of Polyamine and Aminocarboxylate Compounds in Aqueous Solution. Anal. Chem. 1964, 36, 1698-1706.

(23) Johnson, M. P.; Ruban, A. V. Restoration of Rapidly-Reversible Photoprotective Energy Dissipation in the Absence of PsbS Protein by Enhanced $\Delta$ pH. J. Biol. Chem. 2011, 286, 19973-19981.

(24) Teardo, E.; Delaureto, P.; Bergantino, E.; Dallavecchia, F.; Rigoni, F.; Szabo, I.; Giacometti, G. Evidences for Interaction of PsbS with Photosynthetic Complexes in Maize Thylakoids. Biochim. Biophys. Acta, Bioenerg. 2007, 1767, 703-711.

(25) Pan, X.; Li, M.; Wan, T.; Wang, L.; Jia, C.; Hou, Z.; Zhao, X.; Zhang, J.; Chang, W. Structural Insights into Energy Regulation of Light-Harvesting Complex CP29 from Spinach. Nat. Struct. Mol. Biol. 2011, 18, 309-315.

(26) Liu, Z.; Yan, H.; Wang, K.; Kuang, T.; Zhang, J.; Gui, L.; An, X.; Chang, W. Crystal Structure of Spinach Major Light-Harvesting Complex at 2.72 A Resolution. Nature 2004, 428, 287-292.

(27) Jansson, S. A Guide to the Lhc Genes and Their Relatives in Arabidopsis. Trends Plant Sci. 1999, 4, 236-240. 